\title{
Self-Assembly and Intermolecular Forces When Cellulose and Water Interact Using Molecular Modeling
}

\author{
Ali Chami Khazraji and Sylvain Robert \\ Centre de Recherche sur les Matériaux Lignocellulosiques (CRML), Département de Chimie et Physique, \\ Université du Québec à Trois-Rivières, 3351 Boul. des Forges, C.P. 500, Trois-Rivières, QC, Canada G9A 5H7
}

Correspondence should be addressed to Sylvain Robert; sylvain.robert@uqtr.ca

Received 16 January 2013; Revised 14 May 2013; Accepted 16 May 2013

Academic Editor: Jinquan Wei

Copyright (C) 2013 A. Chami Khazraji and S. Robert. This is an open access article distributed under the Creative Commons Attribution License, which permits unrestricted use, distribution, and reproduction in any medium, provided the original work is properly cited.

\begin{abstract}
Cellulose chains are linear and aggregation occurs via both intra- and intermolecular hydrogen bonds. Cellulose has a strong affinity to itself and toward materials containing hydroxyls groups. Based on the preponderance of hydroxyl functional groups, cellulose is very reactive with water. At room temperature, cellulose chains will have at least a monomolecular layer of water associated to it. The formation of hydrogen bonds at the cellulose/water interface is shown to depend essentially on the adsorption site, for example, the equatorial hydroxyls or $\mathrm{OH}$ moieties pointing outward from the cellulose chains. The vdW forces also contribute significantly to the adsorption energy. They are a considerable cohesive energy into the cellulose network. At the surface of the cellulose chains, many intermolecular hydrogen bonds of the cellulose chains are lost. However, they are compensated by hydrogen bonds with water molecules. Electronic clouds can be distorted and create electrostatic dipoles. The large antibonding electron cloud that exists around the glucosidic bonds produces an induced polarization at the approach of water molecules. The electron cloud can be distorted and create an electrostatic dipole. It applies to the total displacement of the atoms within the material. Orbitals play a special role in reaction mechanism. Hydrophilic/hydrophobic nature of cellulose is based on its structural anisotropy. Cellulosewater interactions are exothermic reactions. These interactions may occur spontaneously and result in higher randomness of the system. They are denoted by a negative heat flow (heat is lost to the surroundings). Energy does not need to be inputted in order for cellulose-water interactions to occur.
\end{abstract}

\section{Introduction}

The development of materials derived from renewable resources is an important pillar in the drive for the sustainability of our society. Cellulose is the world's most abundant natural, biodegradable polymer and renewable organic material produced in the biosphere. It is widely distributed in higher plants, in several marine animals, and to a lesser degree in algae, fungi, bacteria, invertebrates, and even amoeba. The production of cellulose is estimated to be over $7.5 \times 10^{10}$ tons/year.

In a woody plant cell, the linear cellulose chains referred to as microfibrils are approximately $3.5 \times 10 \mathrm{~nm}$ in crosssectional dimension and of indeterminate length. Regardless of its source, natural cellulose $\left[\mathrm{C}_{6} \mathrm{H}_{10} \mathrm{O}_{5}\right]_{n} ;(n=10,000$ to 20,000 , where $n$ depends on the cellulose material) can be characterized as a high-molecular-weight homopolymer of $\beta(1 \rightarrow 4)$ linked anhydro-D-glucose monomers organized into a crystalline structure in which every unit is corkscrewed $180^{\circ}$ with respect to its neighbors, and the repeat segment is frequently taken to be a dimer of glucose, known as cellobiose (Figure 1) [1]. Cellulose is composed of $\beta$-D-glucopyranose rings that adopt a ${ }^{4} \mathrm{C}_{1}$ chair conformation, which are linked together by $(1 \rightarrow 4)$-glucosidic bonds [2]. In nature, crystalline cellulose (cellulose I) is found in two forms termed I $\alpha$ and $\mathrm{I} \beta$, and its structure was established in 1984 by crosspolarization magic angle spinning (CP-MAS) $[3,4]$.

Cellobiose $\left(\mathrm{C}_{12} \mathrm{H}_{22} \mathrm{O}_{11}\right)$ is a disaccharide with the formula $\left[\mathrm{HOCH}_{2} \mathrm{CHO}(\mathrm{CHOH})_{3}\right]_{2} \mathrm{O}$. Cellobiose consists of 2 glucose molecules (two D-glucopyranose units) joined by a 1,4,-beta-glycosidic bond. Cellobiose has 8 free alcohol $(\mathrm{OH})$ groups, 1 acetal linkage, and 1 hemiacetal linkage, 


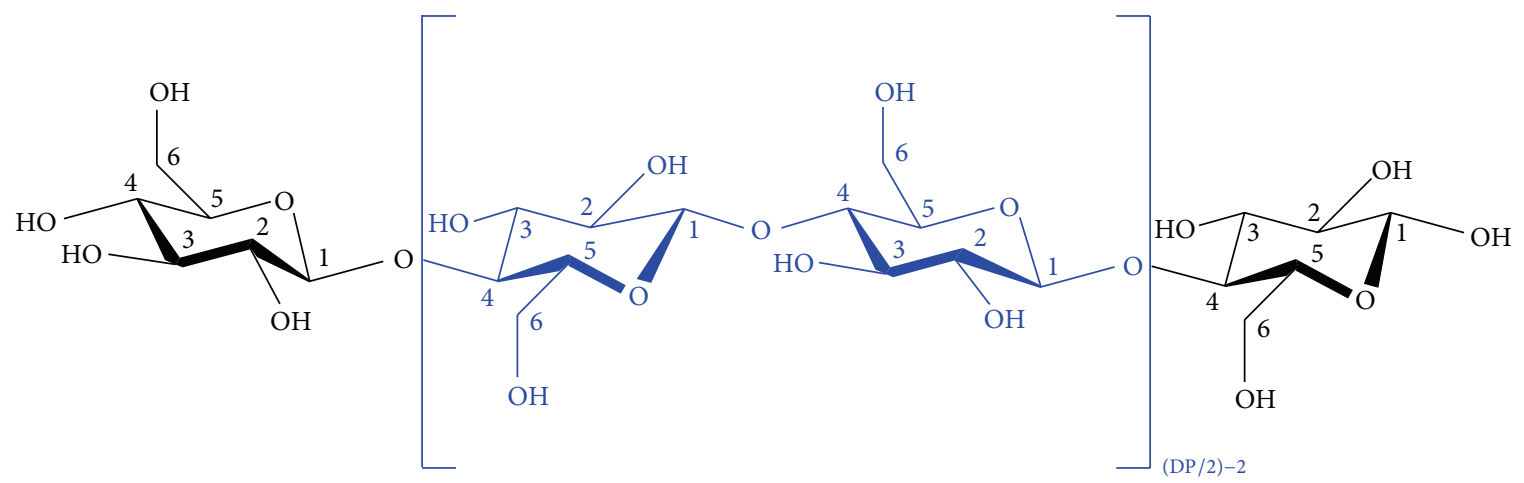

FIGURE 1: Chemical structure of cellulose [1].<smiles>OC[C@H]1O[C@H](O)[C@H](O)[C@@H](O)[C@@H]1O[C@H]1O[C@H](CO)[C@H](O)[C@@H](O)[C@H]1O</smiles>

FIgURE 2: Cellobiose, a 1,4,-beta-glycoside [4-O- $(\beta$-D-glucopyranosyl)- $\beta$-D-glucopyranose].

which give rise to strong inter- and intramolecular hydrogen bonds (Figure 2). Cellobiose cannot be digested by humans and cannot be fermented by yeast. Its solubility in water is $12 \mathrm{~g} / 100 \mathrm{~mL}$. Cellobiose units are reducing sugars because the anomeric carbons on the right-hand sugar are part of a hemiacetal.

Each cellulose chain possesses a directional chemical asymmetry with respect to the termini of its molecular axis: one end is a chemically reducing functionality (i.e., a hemiacetal unit) and the other has a pendant hydroxyl group, the nonreducing end. The number of glucose units or the polymerization degree (DP) is up to 20,000, but shorter cellulose chains can occur and are mainly localized in the primary cell walls [1]. The cellulose polysaccharide contains considerable energy stored in its $\mathrm{C}-\mathrm{H}$ and $\mathrm{C}-\mathrm{C}$ bonds. Cellulose structure favors organization in bundles with the crystalline order held together by hydrogen bonds and disordered regions or amorphous.

In nature, cellulose is mainly found as slender fibrils where a precise number of glucans are organized in a parallel fashion. The lateral size of these fibrils, and consequently the number of its constituting glucan chains, depends on the synthesizing organisms. Extreme fibril lateral dimensions ranging from 2 to $20 \mathrm{~nm}$ have been reported in rare species, but the most common cellulose fibrils have diameters in the 3 to $5 \mathrm{~nm}$ range. Usually, these fibrils do not occur individually but are hierarchically assembled into bundles, herein referred to as fibril aggregates. Furthermore, the fibril aggregates themselves are organized into larger structures. The organization of the glucan chains within each cellulose fibril leads to a specific crystalline arrangement, defined as cellulose I or native cellulose, where all of the glucan chains are crystallized with their polar heads pointing in the same direction. Two crystalline cellulose I allomorphs have been identified, namely, the triclinic cellulose I $\alpha$ and the monoclinic $\mathrm{I} \beta$, the former being dominant in some algal cell walls such as those of Valonia and the latter being the major constituent of higher plant cell walls such as those of cotton or wood [5].

Understanding the supramolecular structure of cellulose and the nature of its overall existing interfaces with water is important in many applications. For chemists working with cellulose, its reactivity is a crucial issue, and this is intimately connected to interfacial properties. Cellulose and its derivatives show a notable variation in their capacity to form anisotropic solutions depending on the kind, the degree of substitution, and the solvent. Molecular structures of typical repeat units of cellulose can be found in the book of Fengel and Wegener [6].

\section{Objective of This Study}

This study will focus on physical and chemical properties of cellulose when it comes into contact with water. We will investigate the crystal structure of cellulose, its electronic properties, and its interactions with water molecules using molecular modeling simulation.

\section{Computational Methods}

The proposal aims to understand some of the fundamentals behind the properties as well as evaluating their stability 


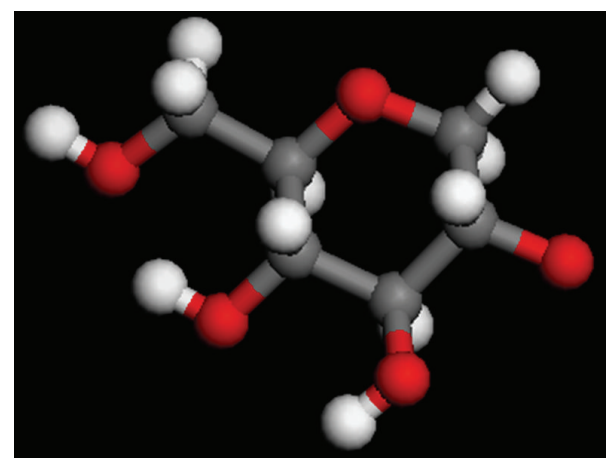

Figure 3: 12aD_glucose molecule.

using mesoscale modeling of cellulose-water interactions. Mesoscale modeling such as the dissipative particle dynamics and the quantum mechanical modeling to be undertaken in this project are prime candidates for tackling the previous questions. The large size and slow dynamics of mesoscale structures make atomistic modeling techniques such as quantum mechanics or molecular mechanics prohibitively expensive. However, mesoscale structures are critical to the properties of certain materials, and therefore methods have been developed to elucidate mesophase behaviour. At such scales, hydrodynamical behaviour becomes important and must be included in the model.

Dissipative particle dynamics (DPD) is a mesoscalesimulation program available from the Accelrys Materials Studio 6.0 [7]. It provides a dynamics algorithm, which incorporates hydrodynamics, for studying coarse-grained systems over long length and time scales. It also enables us to perform analysis on the results of such dynamical simulations. The effective pair potentials are both short ranged and soft, allowing long dynamical time steps to be used. Since the DPD equation of state does not include cubic density terms, the potential does not produce a van der Waals loop in the pressure. As a result, liquid and vapor phases cannot coexist and only a single fluid phase is obtained. The softness of the potential precludes crystalline phases. The walls of cellulose can be included to study preferential adsorption, and the system may be sheared to observe morphological changes.

$\mathrm{DMol}^{3}$ from Accelrys Materials Studio 6.0 is a modeling program that uses density functional theory (DFT) to simulate chemical processes and predicts properties of materials rapidly and with great accuracy. Because $\mathrm{DMol}^{3}$ predicts processes in gas phase, solution, and solid environments, it is broadly applicable to research problems in chemistry, pharmaceuticals, materials science, and chemical engineering, as well as solid-state physics. Researchers can use the program to investigate the nature and origin of the chemical, electronic, optical, and structural properties of a system without the need of any experimental input. DMol ${ }^{3}$ performs virtual experiments, leading to tremendous savings in costly experiments and shorter developmental cycles. $\mathrm{DMol}^{3}$ achieves its speed and accuracy by using numerical functions on an atom-centred grid as its atomic basis [8-11]. The atomic basis functions are obtained from solving the DFT equations for individual atoms and are hence quite accurate. The high quality of these basis sets minimizes basis set superposition effects and provides an improved description of molecular polarizabilities [12]. The electron density in $\mathrm{DMol}^{3}$ is expanded in terms of multipolar, atomic-centred partial densities. This provides a compact yet highly accurate representation of the electronic density and provides good scaling with a growing system size.

VAMP module from Accelrys Materials Studio 6.0 uses semi-empirical calculations to determine a molecular wave function. The molecular wave function is constructed according to the LCAO (Linear Combination of Atomic Orbital) method in which molecular orbitals are obtained as a linear combination Slater-type atomic orbitals. Semi-empirical methods use Slater functions by evaluating the two-electron integrals via a multiple approximation, rather than an exact calculation. Other features of VAMP are transient state optimization, solvent models, and the calculation of many electronic properties.

\section{Results and Discussion}

4.1. First Part: Self-Assembly of Cellulose Chains and Interaction with Water Molecules. The descriptions of polymer systems start with a model of an ideal macromolecule. A polymer built up of $n$ repeating units (bonds) with length $L$ in a linear fashion can be characterized by its reducing and nonreducing extremities. The bonding phenomenon is the sum of a number of mechanical, physical, and chemical forces that overlap and influence each other. Because both hydrogen bonding interactions and vdW dispersion forces are expected to play important roles in determining the crystal structure of cellulose, the proper inclusion of long-range interactions in the geometric optimization is necessary.

From the DPD and Mesocite Accelrys Materials Studio 6.0 library, we took the carbohydrates $12 \mathrm{aD}$ _glucose (Figure 3). First, with this molecule, we built the repeat unit fragment so-called cellobiose (Figure 4) and then cellulose fibres in which the number of chains is 10 and the length of each chain is constructed with 5 repeating units of cellobiose (Figure 5). In this manner, we obtained a fibrous network of cellulose by forming an anhydroglucose polymer connected by $\beta$-glycosidic links. In the chemical sense, cellulose is alinear polymer formed by cellobiose units (two $\beta$-glucose units). Cellulose polymer is insoluble in water. Its structure is compact and ordered. Ithas a great physical and chemical stability.

The molecular aggregation of cellulose in the wood's cell walls contributes to its unique polymer properties. The aggregation phenomenon occurs primarily via both intraand intermolecular hydrogen bonds. The cellulose chains are arranged in a parallel manner and are organized in sheets stabilized by interchain $\mathrm{OH}-\mathrm{O}$ hydrogen bonds, whereas the stacking of the sheets is stabilized by both vdW dispersion forces and weak $\mathrm{CH}-\mathrm{O}$ hydrogen bonds $[13,14]$ (Figures 6 and 7). As result of the vdW dispersion forces, there is a considerable cohesive energy within the cellulose network. The large antibonding electron cloud that exists around the 


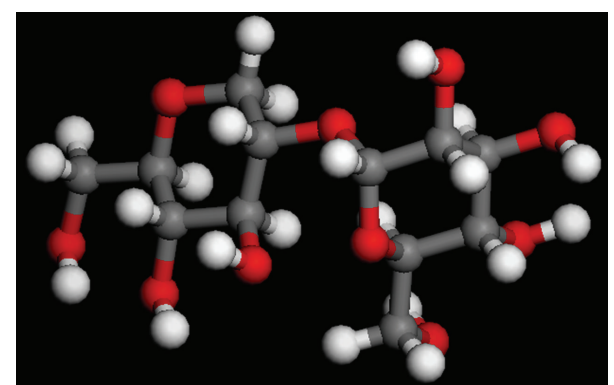

FIGURE 4: Cellobiose unit (12aD_glucose dimer: one repeating unit or cellobiose).

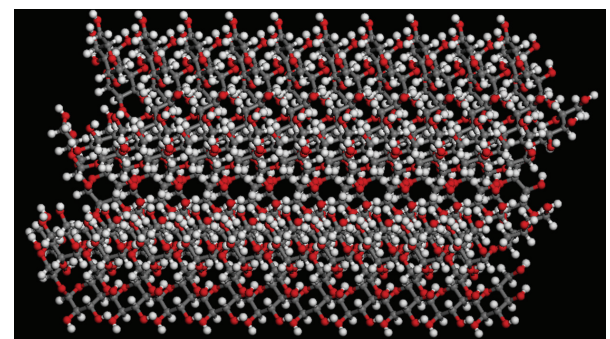

Figure 5: Cellulose chains (chain length: 5 cellobiose units, number of chains: 10 cellulose chains).

glucosidic bonds induces polarization at the approach of the water molecules. The weak interaction due to $\mathrm{CH}-\mathrm{O}$ hydrogen bonds enables the dispersion of cellulose in water in small quantities. Remember that the cellulose chains are interconnected by $\mathrm{OH}-\mathrm{O}$ type hydrogen bonds to form flat chain sheets with weak $\mathrm{CH}-\mathrm{O}$ hydrogen bonds between them. The hydrophilic and hydrophobic nature of cellulose is based on its structural anisotropy. Yamane et al. proposed that the hydrophilic behaviour of cellulose was due to the location of hydroxyl groups at the equatorial positions of the glucopyranose rings [15].

\subsubsection{Description of Interactions between Water Molecules and} Cellulose Chains. Within the plant cell wall matrix, water is subjected to a number of interactions caused by the chemical and physical composition of the cell wall. Thus, the structure and composition of the cell wall produce different states and locations of water, all of which may be important for our understanding of cellulose-water interactions.

The behaviour of cellulose surfaces in different media as well as their interactions with different chemicals is of great importance in their current and future applications (papermaking, composites, and nanocomposites). The bonding phenomenon is the sum of a number of mechanical, physical, and chemical forces that overlap and influence each other. Wetting may be attributed to acid-base interactions, weak hydrogen bonding, or vdW dispersion forces (dipoledipole, orientation, induction and dispersion forces). The extent of wetting depends on the difference in surface free energies of the solid, liquid, and subsequent interface.

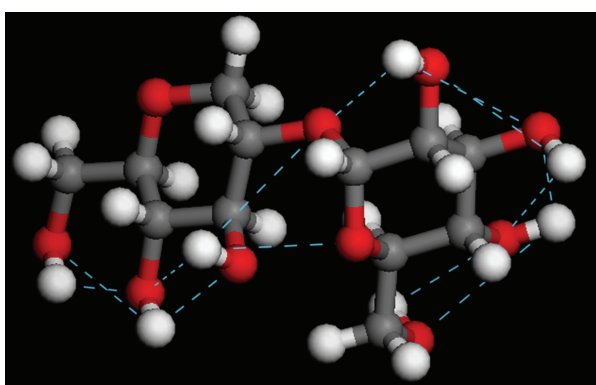

FIGURE 6: Intramolecular hydrogen bonds in the cellobiose unit (blue dashed lines).

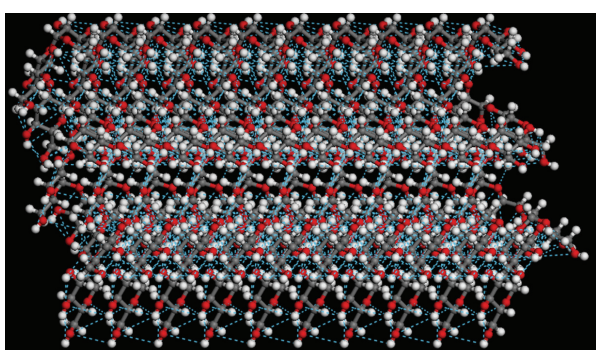

FIGURE 7: Intra- and intermolecular hydrogen bonds in the cellulose chains (chain length: 5 cellobiose units, number of chains: 10 cellulose chains).

Since cellulose has a strong affinity to itself and any hydroxyl-containing materials, especially, water [16], a solvation cage is formed around the cellulose chains after their interactions with water molecules (Figure 8). The cellulose reacts much more with water because of the preponderance of hydroxyl functional groups. In the cellulose model in Figure 7, we added a lot of water molecules. As a result, the water molecules interacted quickly with the cellulose chains by immediately forming hydrogen bonds (Figure 8 , blue dashed lines). According to a previous study, only the topmost layer in the cellulose differs in terms of structure and dynamics from the crystal bulk, but even these differences are small. At the surface approximately half of the cellulose intermolecular hydrogen bonding is lost, but this is compensated by hydrogen bonds with water molecules. The potential energy of water molecules in the first hydration layer is lower by $2 \mathrm{~kJ} / \mathrm{mol}$. The cellulose surface contains about five exposed hydroxyl groups per square nanometer, which accounts for the good hydration of the surface [17]. In addition, monomeric properties of nanocrystalline cellulose offer high specific surface.

Water is a good solvent due to its polarity. The molecular shape of water allows for this polarity. Cellulose polymer is also a polar compound. Therefore, when the cellulose chains come into the water media, these chains are automatically surrounded by water molecules. The smallness of the water molecules enables them to react easily with the cellulosic chains. The partial negative charge of the dipole of water is attracted by the positive charge of the cellulose chains and vice versa. Cellulose chains may be surrounded by several water molecules. At common ambient environment 


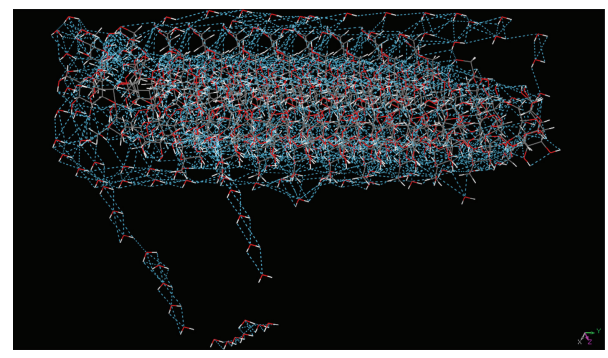

FIGURE 8: Hydrogen bonds (blue dashed lines) between water molecules and cellulose chains (chain length: 5 cellobiose units, number of chains: 10 cellulose chains).

conditions (room temperature), cellulose chains will have at least a monomolecular layer of water associated with it. Moreover, cellulose is very stable in a variety of solvents and can only be dissolved by the application of strong acids or strong hydrogen-bonding-solvent systems-usually aminebased. The thermal properties of cellulose are such that the cellulose glass transition temperature is in the range of 200 to $230^{\circ} \mathrm{C}$, which is close to its thermal decomposition temperature of $260^{\circ} \mathrm{C}$ [18].

The hydrogen bond is really a special case of dipole forces. A hydrogen bond is the attractive force between the hydrogen attached to an electronegative atom of one molecule and an electronegative atom of a different molecule. Usually the electronegative atom is oxygen, nitrogen, or fluorine, which has a partial negative charge. The hydrogen then has the partial positive charge. To recognize the possibility of hydrogen bonding, examine the Lewis structure of the molecule. The electronegative atom must have one or more unshared electron pairs as in the case of oxygen and must have a negative partial charge. The hydrogen, which has a partial positive charge, tries to find another atom of oxygen with excess electrons to share and is attracted to the partial negative charge. This forms the basis for the hydrogen bond. In other words, The hydrogen on one molecule attached to $\mathrm{O}$ that is attracted to an $\mathrm{O}$ of a different molecule.

\subsubsection{Building a Cellulose Crystal Containing Water Molecules} inside. Accelrys Materials Studio 6.0 features a powerful crystal-building tool which enables building the cellulose crystal. The display style of the water atoms changes to large CPK (Corey-Pauling-Koltun) spheres with their radii dependent on the vdW radius of the element they represent. However, most structures look better with a slightly smaller CPK size, so that the spheres do not dominate the structure too much.

Change the range values so that more than one cell is displayed. Three cells are displayed in the $x$-direction $(A=3)$, and then we increased the $\mathrm{B}$ and $\mathrm{C}$ values similarly $(\mathrm{B}=$ $3, \mathrm{C}=5$ ). The cells are extended in all three directions. The crystals simulated are either isolated or immersed in water. Using the $12 \mathrm{aD}$ _glucose dimer (Figure 9), we built the cellulose crystal in which there are water molecules (Figure 10). Within the cellulose chains, the water molecules are shown in large spheres or CPK style and cellulose chains

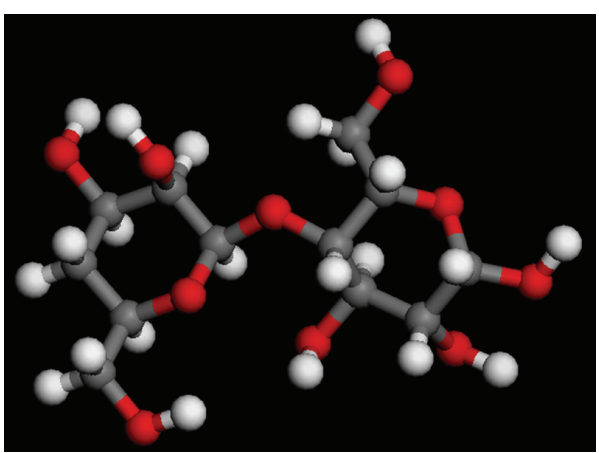

Figure 9: Cellobiose unit (12aD_glucose dimer).

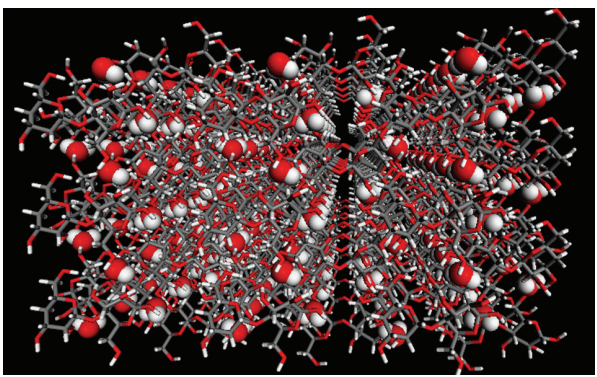

FIGURE 10: Extended structure of cellulose chains (stick) and water molecules shown in CPK style (range $\max \mathrm{A}=3, \mathrm{~B}=3, \mathrm{C}=5$ ) before interactions between them.

are displayed in stick. For larger structure such as cellulose polymer that we are visualizing, the perspective projection is useful. Water molecules interact with the cellulose chains and by creating hydrogen bonds (Figure 11). Through the capillary forces, water molecules can be found around the fibres, for example, the diffusion of water molecules in porous fibre network in order to understand the permeability to liquids.

The bonding phenomenon is the sum of a number of mechanical, physical, and chemical forces that overlap and influence each other. In addition, water molecules tend to congregate in forming a bulk of hydrogen bonds or clusters (Figures 12 and 13). This bulk does not also prevent the water molecules from binding with cellulose chains. The water could form different kinds of shapes, especially, clusters with the number of water molecules.

4.2. Second Part: Some Physical and Chemical Properties of Interactions between Cellulose and Water. In this section, we tried to highlight some energetic and electronic properties of cellulose using $\mathrm{DMol}^{3}$ from Accelrys Materials Studio 6.0. The purpose is to calculate binding energies and ionization potentials because, between cellulose and water, there are electronic interactions.

4.2.1. Effect of Water Adsorbates on the Field Emission from Cellobiose Tips. Of the various potential application areas of cellulose, field-emission-based flat panel display is the closest to becoming the first commercial application. In these devices, electrons are emitted from tips of cellobiose, which 


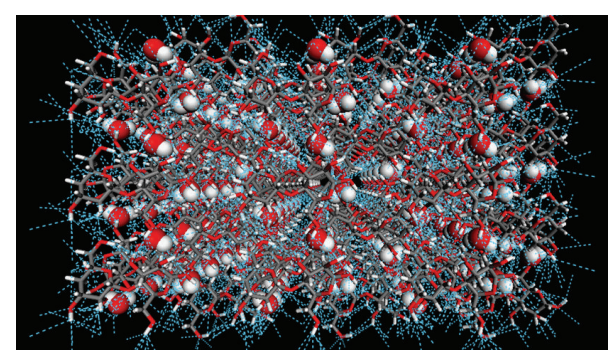

FIGURE 11: Extended structure of cellulose chains (stick) and water molecules shown in CPK style ( range $\max \mathrm{A}=3, \mathrm{~B}=3, \mathrm{C}=5$ ) after interactions between cellulose chains and water molecules with hydrogen bonds (blue dashed lines).

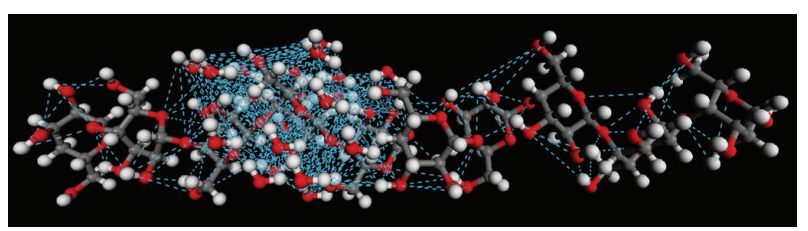

FIGURE 12: Interactions between one cellulose chain (containing 5 cellobiose units) and water molecules (19 molecules).

have been aligned parallel on silicon or glass substrates. A practical challenge in making an efficient display is to reduce the operating voltage under which electron emission occurs. One way to achieve this is to introduce adsorbates that might effectively lower the ionization potential (IP) and facilitate the extraction of electrons from the cellobiose tip. Important experiments in this context were recently performed at Motorola [19], showing that the presence of water significantly enhances the field emission current from cellobiose.

In this experiment, we will use first-principles quantum mechanics to compute the binding energy of a water cluster to a cellobiose tip under field emission conditions. We will also compute the ionization potential, a measure of the difficulty to extract an electron from the cellobiose tip. The Accelrys Materials Studio 6.0 module used to do this calculation is $\mathrm{DMol}^{3}$ [20], a first-principles code based on the density functional theory (DFT). In this code, the electronic wave functions are expanded in an atom-centered basis set defined on a numerical grid.

We have built the preoptimized structures of

(1) the isolated cellobiose tip (tip.xsd), where only the repeat segment is frequently taken to be a dimer of glucose, known as cellobiose (Figure 14);

(2) an H-bonded cluster of 5 water molecules (5water .xsd) (Figure 15); and

(3) the cellobiose tip with the water cluster adsorbed on it (tip_5water.xsd) (Figure 16).

In the first structures (tip.xsd and tip_5water.xsd), the axis is aligned along the negative $Z$-direction. In order to simulate field emission conditions, calculations (1) and (3) are

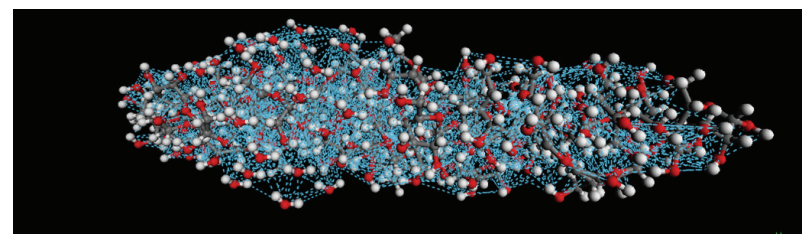

FIGURE 13: Interactions between four cellulose chains (each chain containing 5 cellobiose units) and water molecules (19 molecules).

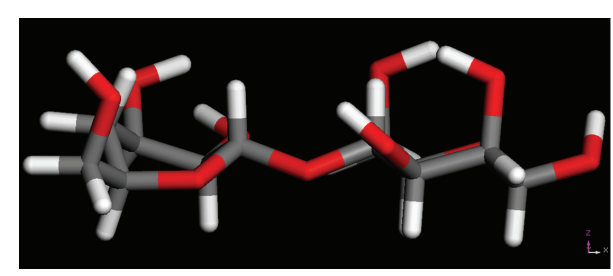

FIGURE 14: Cellobiose tip (tip.xsd).

performed in an electric field oriented along the positive $Z$ axis, while calculation (2) is performed in zero electric field (see [21] for more details).

This modeling covers

(i) computing the single point energy for the cellobiose tip + water system;

(ii) computing the single point energy for the cellobiose tip;

(iii) computing the single point energy for the water system in the absence of an electric field;

(iv) computing the binding energy of water to the cap;

(v) ionization potential (IP) calculations.

(1) Computing the Single Point Energy for the Cellobiose Tip + Water System

On tip_5water.xsd that it is the activated model.

On the setup tab:

task is set to energy and functional to GGA and PBE.

On the electronic tab:

integration accuracy is coarse.

SCF tolerance is coarse, and basis set to MIN.

On the $\mathrm{DMol}^{3}$ electronic options dialog, in the SCF tab, check use smearing. On the orbital cutoff tab custom is selected. In the global orbital cutoff textbox 3.5 is indicated.

In the tip_5water.input file, we erased the line symmetry on, and we added the following lines in the document: symmetry $\mathrm{cl}$ fix and electric field 0.00 .0 0.02 .

The symmetry line is to make sure that $\mathrm{DMol}^{3}$ does not reorient the molecule away from the current alignment along the $Z$-direction. The electric field units are in Hartree/Bohr, which implies a field of 


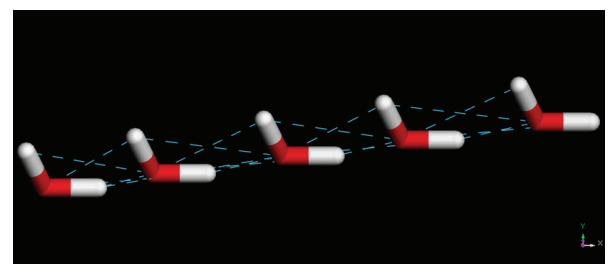

FIGURE 15: Water system (5water.xsd).

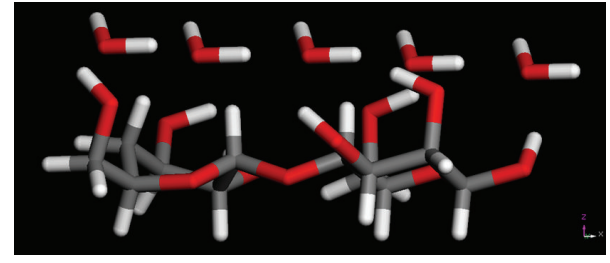

FIGURE 16: Cellobiose and water system (tip_5water.xsd).

$\sim 1$ volt/Angstrom parallel to the tube axis directed toward the cellobiose tip from above.

Once the calculation finishes, in the tip_5water.outmol file, the total energy $\left(E_{f}\right)$ is

$$
E_{\text {tip_5water }}=-1600.540 \mathrm{Ha} \text {. }
$$

\section{(2) Computing the Single Point Energy for the Cellobiose Tip}

With tip.xsd as the activated model, use the same settings in the calculation dialog of $\mathrm{DMol}^{3}$ as tip_5water.xsd.

Again, the "symmetry" line is to make sure that $\mathrm{DMol}^{3}$ does not reorient the molecule away from the current alignment along the $Z$-direction.

Once the calculation finishes, the total energy $\left(E_{f}\right)$ is

$$
E_{\text {tip }}=-1219.746 \mathrm{Ha} \text {. }
$$

(3) Compute the Single Point Energy for the Water System in the Absence of Electric Field

Again, with 5water.xsd as the activated model, use the same settings in the calculation dialog of $\mathrm{DMol}^{3}$ as tip_5water.xsd.

After the job finishes, the total energy $\left(E_{f}\right)$ is

$$
E_{5 \text { water }}=-381.219 \mathrm{Ha} \text {. }
$$

(4) Compute the Binding Energy of Water to the Tip

The binding energy is given by the formula

$$
\begin{aligned}
E_{\text {tip }}+E_{5 \text { water }}-E_{\text {tip_5water }}= & -1219.746 \\
& +(-381.219)-(-1600.540)
\end{aligned}
$$

$$
\begin{aligned}
& =-0.425 \mathrm{Ha} \\
& =-266.692 \mathrm{kcal} / \mathrm{mol}
\end{aligned}
$$

$(1$ hartrees $=627.51 \mathrm{kcal} / \mathrm{mol})$.

\section{(5) Ionization Potential (IP) Calculations}

In order to obtain insight into the effect of adsorbed water molecules on the field emission current, it is interesting to compute the ionization potential (IP) of an electron from the cellobiose tip in the presence and absence of water.

The IP is defined as the difference in energy between a system with a +1 charge (i.e., 1 electron removed) and the original system with a net zero charge. Also, the system with the +1 charge has essentially the same atomic geometry as the neutrally charged system flowing in from the stem side of the cellobiose. Thus all we need to perform is the single point energy with the same $\mathrm{DMol}^{3}$ settings as before, except that charge has to be set to +1.0 .

On the tip_5water.input file, we changed the charge from 0.0 to 1.0 .

The energy for the +1 charged system is

$$
E_{f}=-1600.332 \mathrm{Ha} .
$$

On the tip.input file, we changed the charge from 0.0 to 1.0 .

The total energy of the +1 charged cellobiose tip is

$$
E_{f}=-1219.458 \mathrm{Ha} \text {. }
$$

The IP for the cellobiose tip (in absence of water) is therefore

$$
1219.746-1219.458=0.288 \mathrm{Ha} .
$$

In the presence of water, the IP becomes

$$
1600.540-1600.332=0.208 \mathrm{Ha} .
$$

So, the presence of water cluster lowers the IP by $0.289-0.225=0.064 \mathrm{Ha}$.

$0.288-0.208=0.080 \mathrm{Ha}=50.068 \mathrm{kcal} / \mathrm{mol}$, thereby enhancing field emission current at a given voltage.

4.3. Third Part: Electronic Properties of Cellulose and Water. In this modeling, we optimized the cellobiose and water molecules, analyzed the results, and calculated molecular properties using $\mathrm{DMol}^{3}$ from Accelrys Materials Studio 6.0. We built an atomistic model of cellobiose and water (Figure 17), and then we modeled them using quantum mechanics. 


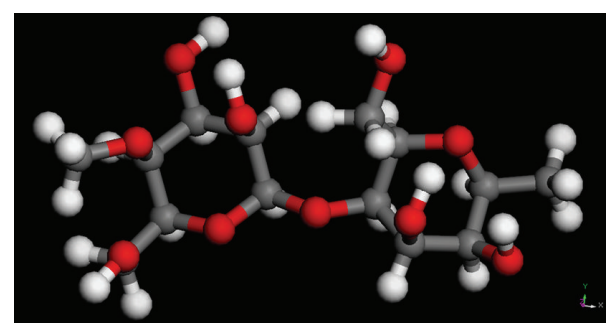

FIGURE 17: Cellobiose unit (repeating unit of the cellulose chains).

Since cellulose readily interacts with its environment and in a special way with water molecules, it is best to highlight its electronic properties as the electron density, electrostatic potential field, and molecular orbitals where the reactions take place.

In the $\mathrm{DMol}^{3}$ calculation, in the setup tab, we have set the task to geometry optimization. The quality is set to medium, the functional to GGA (generalized gradient approximation) and BLYP $[22,23]$. We have just specified the basis set and the Hamiltonian to be used in this calculation. In the electronic tab, we have set the basis set to DNP (double numerical plus polarization). In the properties tab, we have checked the electron density, electrostatics, and orbitals.

\subsubsection{Displaying the Electronic Properties of Cellulose Chains} and Water Molecules. We used the $\mathrm{DMol}^{3}$ analysis tool from Accelrys Materials Studio 6.0 to display isosurfaces of cellobiose and water molecules. Initially, we are going to view the electron density as an isosurface. The total charge density is displayed. Once we have an isosurface displayed, we can map different properties onto it, such as the electrostatic potential of cellobiose and water molecules (Figures 18 and 19, resp.). The electrostatic potential is mapped onto the total charge density isosurface using color to represent the variation. Mapping the electrostatic potential in this manner gives insight into the charge distribution over the molecule.

Electrostatic potential:

(i) positive regions (red) correspond to electron-deficient areas-subject to nucleophilic attack;

(ii) negative regions (blue) correspond to electron-rich areas-subject to electrophilic attack.

We used VAMP from Accelrys Materials Studio 6.1 for these experiments. WAMP is a semiempirical molecular orbital program that has been optimized to be highly numerically stable. We optimized the structures of a cluster of water (4 water molecules), cellobiose, and cellobiose +4 water molecules, and we analyzed several properties of theirs (Table 1). Polar molecules, such as water molecules, have a weak, partial negative charge at one region of the molecule (the oxygen atom in water) and a partial positive charge elsewhere (the hydrogen atoms in water). Thus when water molecules are close together, their positive and negative regions are attracted to the oppositely charged regions of nearby molecules. The force of attraction, shown here as blue dashed lines, is called a hydrogen bond (Figure 20).

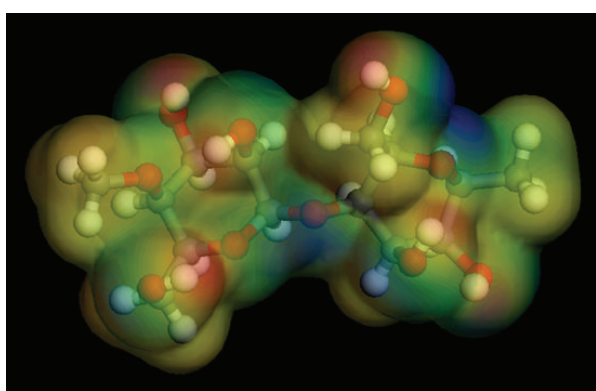

FIGURE 18: Electrostatic potential field added on electron density of cellobiose unit.

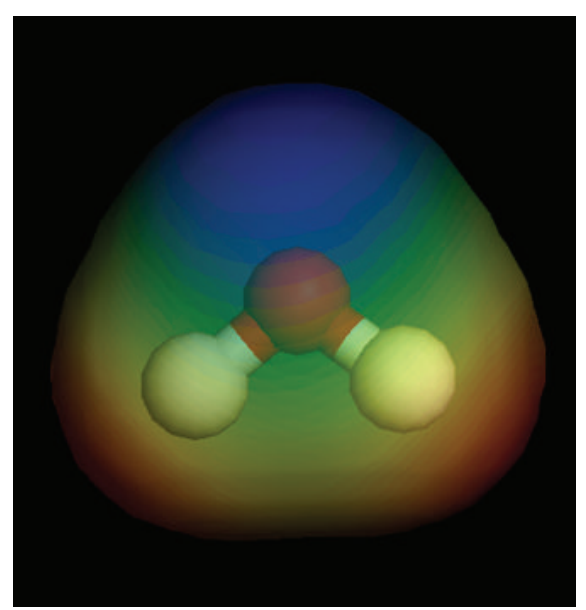

FIGURE 19: Electrostatic potential field added on electron density of water molecule.

Each water molecule is hydrogen bonded to four others. The hydrogen bonds that form between water molecules account for some of the essential and unique properties of water. The graphic shows a cluster of water molecules in the liquid state (Figure 20). Water is a polar molecule, with the oxygen (red) being the negative area and the hydrogen (white) being the more positive area. Opposite charges attract. The bond lengths give some indication of the bond strength. A normal covalent bond is 0.96 Angstroms, while the hydrogen bond length is $1.97 \AA$. The molecular electrostatic potential is the potential energy of a proton at a particular location near a molecule. Negative electrostatic potential corresponds to an attraction of the proton by the concentrated electron density in the molecules (from lone pairs, pi-bonds, etc.). Positive electrostatic potential corresponds to repulsion of the proton by the atomic nuclei in regions where low electron density exists and the nuclear charge is incompletely shielded. The polarity of the water molecule with the attraction of the positive and negative partial charges is the basis for the hydrogen bonding.

We calculated several properties when cellulose and water interact shown in Table 1. Many chemical reactions release energy in the form of heat, light, or sound. Cellulose-water interactions are exothermic reactions. Exothermic reactions may occur spontaneously and result in higher randomness 
TABLE 1: Heat of formation and electronic properties of cellulose-water interactions.

\begin{tabular}{lccc}
\hline & Four water molecules & Cellobiose & Cellobiose +4 water molecules \\
\hline Heat of formation $\left(\mathrm{kcal} \cdot \mathrm{mol}^{-1}\right)$ & -260.88 & -491.31 & -753.73 \\
Electron energy $(\mathrm{eV})$ & -3903.2 & -35114.2 & -50193.8 \\
Core-core repulsion $(\mathrm{eV})$ & 2507.9 & 30066.9 & 43751.1 \\
Total energy $(\mathrm{eV})$ & -1395.3 & -5047.3 & -6442.6 \\
Ionization potential $(\mathrm{eV})$ & 12.2 & 10.8 & 10.3 \\
Molecular weight $(\mathrm{D})$ & 72.061 & 326.30 & 398.361 \\
\hline
\end{tabular}

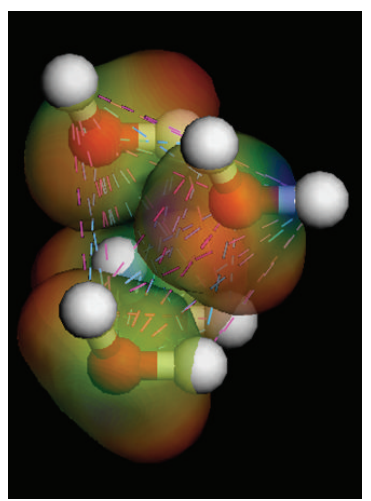

(a)

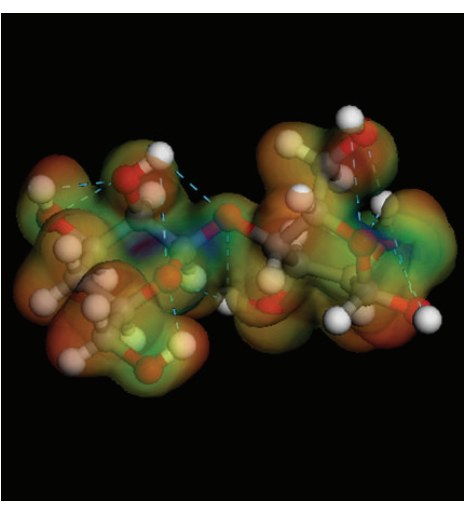

(b)

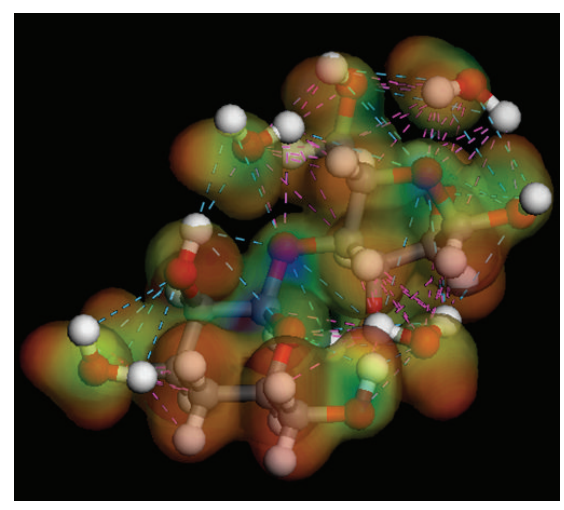

(c)

FIGURE 20: Electrostatic potential mapped onto the total charge density isosurface of 4 water molecules, cellobiose, and cellobiose +4 water molecules, respectively, with hydrogen bonds (blue dashed lines) and vdW (pink dashed lines).

of the system. They are denoted by a negative heat flow (heat is lost to the surroundings). In the lab, exothermic reactions produce heat or may even be explosive. Cellulosewater interactions describe a process that releases energy in the form of heat. Forming chemical bonds releases energy and therefore is an exothermic process. Energy does not need to be inputted in order for cellulose-water interactions to occur.

The heat of formation for the cluster of water (4 water molecules), cellobiose, and cellobiose +4 water molecules, as calculated by WAMP, is approximately $-260.88 \mathrm{kcal} / \mathrm{mol}$, $-491.31 \mathrm{kcal} / \mathrm{mol}$, and $-753.73 \mathrm{kcal} / \mathrm{mol}$, respectively. Examples for other properties displayed are the total electronic and the total nuclear repulsion energies. The difference between these two energies gives the total energy. The next two lines contain two convergence criteria, the gradient norm and the root-mean-square force on the atoms. Default convergence criteria for these are $0.01 \mathrm{Ha}$ and $0.4 \mathrm{kcal} / \mathrm{mol}$. The output shows that both convergence criteria are satisfied. Finally, the ionization potential, the number of filled molecular orbitals, and the molecular weight of 4 water molecules, cellobiose, and cellobiose +4 water molecules are listed.

\subsubsection{Modeling Interactions between Cellulose and Water} Molecules Using Quantum Mechanics. We used $\mathrm{DMol}^{3}$ tool from Accelrys Materials Studio 6.0 to display electronic properties after interactions between cellulose chains and water molecules. The cellulose chains are very reactive with water molecules. Hydrogen bonds form as soon as the cellulose chains come in contact with water molecules (Figure 21). Hydrogen bonding is often described as an electrostatic dipole-dipole interaction. Electronic density and electrostatic potential fields are mixed up between cellulose chains and water molecules. When cellulose and water interact, the electron clouds surrounding each atom of cellulose and water are combined and they form a single electron cloud. Consequently, cellulose chains and water molecules become a single entity (Figures 22 and 23).

Cellulose chains and water molecules can interact through vdW dispersion forces, hydrogen bonding, stacking $\pi$ electrons, and charge-transfer interactions. The $\mathrm{vdW}$ dispersion forces are electrostatic types. They are born from attraction between the dipoles of water molecules and cellulose chains. The attraction forces between the dipoles are lowest in the order of a few $\mathrm{kcal} / \mathrm{mol}(<10)$. When water molecules come into cellulose chains, it results in an electrostatic attraction between the hydrogen atom carrying a $\delta+$ charge and an electronegative atom of another cellulose chain carrying a $\delta$ - charge. Moreover, hydrogen bonds are formed whenever a hydrogen atom is linked to a very electronegative atom. It is both a hydrogen-bond donor and hydrogen-bond acceptor or a hydrogen-bond acceptor and hydrogen-bond donor. It reaches energy of around $10 \mathrm{kcal} / \mathrm{mol}$. The available bond around an atom depends on the arrangement of electron pairs in the valence layer. The arrangement depends on the number of electron pairs. The most likely arrangement 


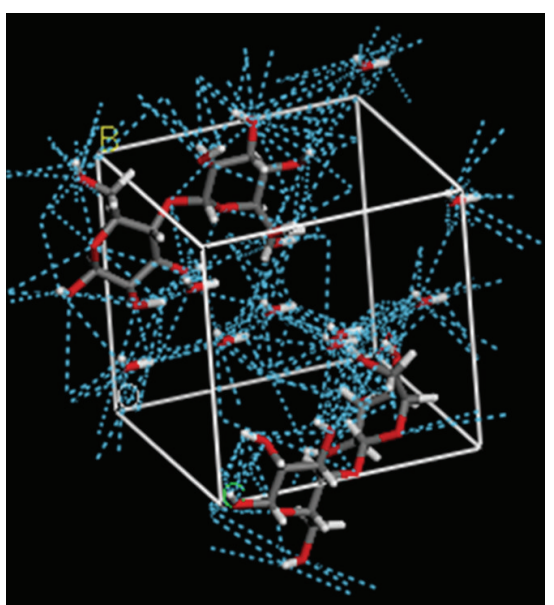

FIGURE 21: Interactions between cellulose chains and water molecules showing hydrogen bonds between them (blue dashed lines).

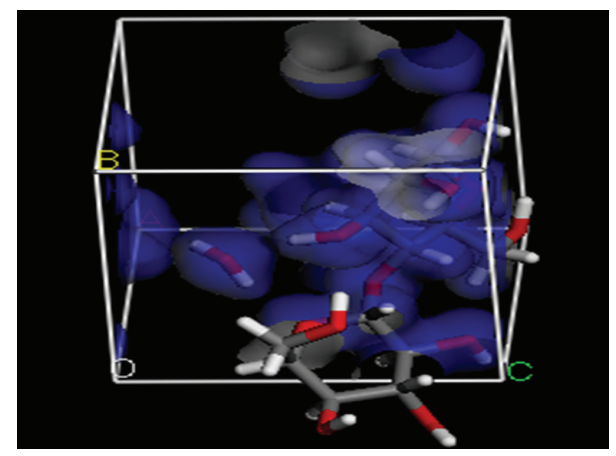

FIGURE 22: Electron density when cellulose chains and water molecules interacted.

is that which minimizes the electronic repulsion, that is, one that separates the maximum electron pairs.

Detailed knowledge from the atomic and electronic levels is of direct scientific interest and provides insight into understanding the interaction/reaction mechanism of much more complex systems. According to the LCAO theory, when water molecules and cellulose chains come together, we can understand the new molecular orbitals (and their energies) in terms of the orbitals (and energies) of the separated species. The interactions of cellulose orbitals of one species with the other orbitals of water generate two new orbitalsone favorable (bonding) combination, which is lower in energy than either of the separate orbitals, and the other an unfavorable (antibonding) combination, which is higher than either of the separated orbitals. The amount by which the energies shift depends on overlap and energy match between the mixing orbitals.

Lewis theory (1916) says that two or more atoms react to give a chemical bond if the entity obtained is more stable (a combination accompanied by a release of energy). The establishment of a chemical bond affects only the outer

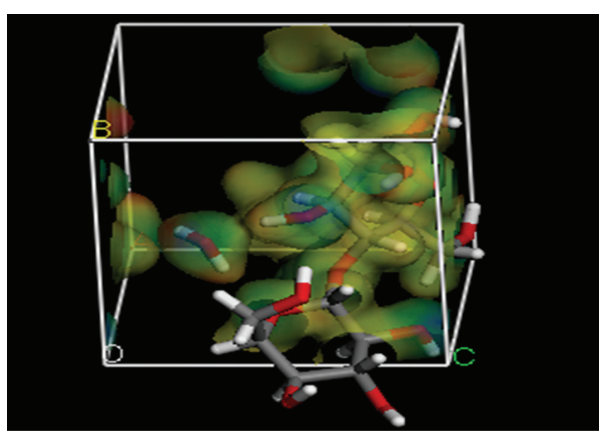

FIGURE 23: Electrostatic potential field when cellulose chains and water molecules interacted.

electrons-valence electrons. Electrons in deep layers are not affected.

As known, a water molecule is formed when two atoms of hydrogen bond covalently with an atom of oxygen. In a covalent bond, electrons are shared between atoms. In water, the sharing is not equal. The oxygen atom attracts the electrons more strongly than the hydrogen. This gives water an asymmetrical distribution of charge. Molecules that have ends with partial negative and positive charges are known as polar molecules. It is this polar property that allows water to separate polar solute molecules and explains why water can dissolve so many substances. Water is a good solvent due to its polarity. The solvent properties of water are vital in biology because many biochemical reactions take place only within aqueous solutions. When an ionic or polar compound enters water, it is surrounded by water molecules. The relatively small size of water molecules typically allows many water molecules to surround one molecule of solute. The partially negative dipoles of the water are attracted to positively charged components of the solute and vice versa for the positive dipoles.

In this experiment, we used VAMP to calculate the Mulliken charges and bond orders of cellobiose and water molecule. The partial atomic charges, as calculated by the Coulson method, are displayed on the cellobiose and water molecule (Figures 24 and 25, resp.). Mulliken charges arise from the Mulliken population analysis and provide a means of estimating partial atomic charges from calculations carried out by the methods of computational chemistry, particularly those based on the LCAO method. The bonds in the cellobiose and water molecule are updated from the VAMP calculation (Figures 26 and 27, resp.).

To calculate the electronic properties of cellulose unit and one water molecule, we used VAMP from Accelrys Materials Studio 6.0. This tool enabled us to calculate the heat of formation of these two reactive entities and other important molecular properties (Table 2).

The heat of formation for cellobiose unit and for one water molecule, as calculated by VAMP, approximately $-487.66 \mathrm{kcal} / \mathrm{mol}$ and $-59.24 \mathrm{kcal} / \mathrm{mol}$, respectively. The difference between the total electronic energy and the total nuclear repulsion energy (core-core repulsion) energies gives the total energy. 


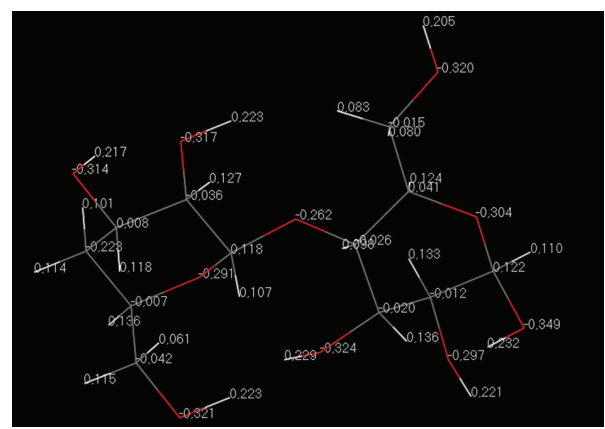

FIGURE 24: Mulliken charges of cellobiose unit.

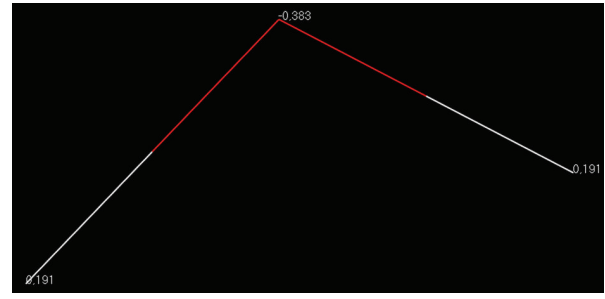

FIGURE 25: Mulliken charges of water molecule.

\section{Conclusion}

Molecular aspects of the interactive behaviour between cellulose and water are not well known. Molecular modeling simulations are one of the most convenient tools to predict solvent properties of cellulose-water interactions, which are otherwise difficult to predict or measure by experimental means. Cellulose is the most abundant chemical compound on earth, and its natural affinity for self-adhesion has long been recognized. The ease of adhesion that occurs in cellulose has contributed to its use in paper and other fibre-based composite materials.

It is obvious that the structure of cellulose is complex, and its study requires the consideration of many factors. Investigation into the many aspects of the cellulose structure is being continued by research groups worldwide and new results constantly appear in the literature. This trend shall continue as more is learned about cellulose by the application of an increasing number of techniques including molecular modeling and simulation solutions. All knowledge gained about cellulose structure is vital to life today because cellulose and its derivatives are used in so many industrial applications.

Within the plant cell wall matrix, water molecules are subjected to a number of interactions caused by the chemical and physical composition of the cell wall. Structure and composition of the cell wall produce different states and locations for water. Besides a strong affinity to itself, the cellulose has also a strong affinity toward materials containing hydroxyl groups, especially water. Cellulose chains were inter-connected by $\mathrm{OH}-\mathrm{O}$ type hydrogen bonds to form flat sheets with $\mathrm{CH}-\mathrm{O}$ hydrogen bonds between them. After the interactions between cellulose chains and water molecules, many intermolecular hydrogen bonds are broken from the cellulose/water interface, but these chains build new

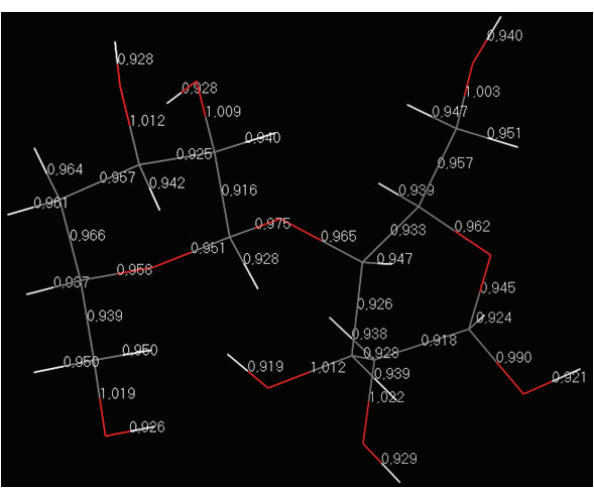

FIgURE 26: Bond order of cellobiose unit.

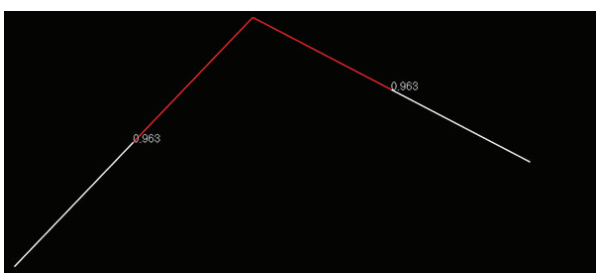

FIGURE 27: Bond order of water molecule. connections with the water molecules. The weak interactions also allow the dispersion of cellulose in water even in small quantities. The partial negative charge of the dipole water is attracted by the positive charge of the cellulose and vice-versa.

The bonding phenomenon is the sum of a number of mechanical, physical and chemical forces that overlap and influence each other. Within the plant cell wall matrix, water is subjected to a number of interactions caused by chemical and physical composition of the cell wall. The structure and composition of the cell wall produce different states and locations of water. Cellulose chains can interact through hydrogen bonds, vdW forces, stacking $\pi$ and charge transfer interactions. The vdW is electrostatic forces type. They arise between dipoles. The topmost surface layer of the nanocrystalline cellulose is structurally affected by the water outside the surface, which reflects the rigidity and strength of the cellulose crystal. Cellulose chains are arranged in a parallel manner and organized in sheets stabilized by interchain $\mathrm{OH}-\mathrm{O}$ hydrogen bonds, whereas the stacking of the sheets is stabilized by both vdW forces and weak $\mathrm{CH}-\mathrm{O}$ hydrogen bonds. Cellulose chains were inter-connected by $\mathrm{OH}-\mathrm{O}$ type hydrogen bonds to form flat sheets with weak $\mathrm{CH}-\mathrm{O}$ hydrogen bonds.

\section{Future Work and Perspective}

(i) The extent of wetting depends on the difference in surface free energies of the solid, liquid and subsequent interface.

(ii) In addition of non-bonding interactions, wetting may be attributed to acid-base interactions. 
TABLE 2: Heat of formation and electronic properties of cellobiose unit and water molecule.

\begin{tabular}{lcc}
\hline & Cellobiose unit & Water molecule \\
\hline Heat of formation $\left(\mathrm{kcal} \cdot \mathrm{mol}^{-1}\right)$ & -487.66 & -59.24 \\
Electronic energy $(\mathrm{eV})$ & -35393.3 & -493.3 \\
Core-core repulsion $(\mathrm{eV})$ & 30346.2 & 144.7 \\
Total energy $(\mathrm{eV})$ & -5047.1 & -348.6 \\
Ionization potential $(\mathrm{eV})$ & 10.5 & 12.5 \\
Molecular weight $(\mathrm{D})$ & 326.30 & 18.015 \\
\hline
\end{tabular}

(iii) Cellulose-water interactions are exothermic reactions. These interactions may occur spontaneously and result in higher randomness or entropy $(\Delta S>0)$ of the system. They are denoted by a negative heat flow (heat is lost to the surroundings) and decrease in enthalpy $(\Delta H<0)$. Energy does not needs to be inputted in order for cellulose-water interactions to occur.

\section{Acknowledgments}

The authors would like to thank NSERC (National Sciences and Engineering Research Council of Canada), the Innovative Green Wood Fibre Products Network, and the CQMF (Centre québécois sur les matériaux fonctionnels) for financial support. They also thank the Compute Canada network and Calcul Québec for their computational resources.

\section{References}

[1] Y. Habibi, L. A. Lucia, and O. J. Rojas, "Cellulose nanocrystals: chemistry, self-assembly, and applications," Chemical Reviews, vol. 110, no. 6, pp. 3479-3500, 2010.

[2] E. Sjöström, Wood Chemistry: Fundamentals and Applications, Academic Press, San Diego, Calif, USA, 1981.

[3] R. H. Atalla and D. L. VanderHart, "Native cellulose: a composite of two distinct crystalline forms," Science, vol. 223, no. 4633, pp. 283-285, 1984.

[4] D. L. VanderHart and R. H. Atalla, "Studies of microstructure in native celluloses using solid-state 13C NMR," Macromolecules, vol. 17, no. 8, pp. 1465-1472, 1984.

[5] M. Bergenstråhle, J. Wohlert, P. T. Larsson, K. Mazeau, and L. A. Berglund, "Dynamics of cellulose-water interfaces: NMR spin-lattice relaxation times calculated from atomistic computer simulations," Journal of Physical Chemistry B, vol. 112, no. 9, pp. 2590-2595, 2008.

[6] D. Fengel and G. Wegener, Wood: Chemistry, Ultrastructure, Reactions, Walter de Gruyter, New York, NY, USA, 1983.

[7] Accelrys Software Inc, Material Studio Modeling Environment, Release 6, Accelrys Software Inc, San Diego, Calif, USA, 2011.

[8] B. Delly, "An all-electron numerical method for solving the local density functional for polyatomic molecules," Journal of Chemical Physics, vol. 92, no. 1, article 508, 1990.

[9] B. Delly, "Analytic energy derivatives in the numerical localdensity-functional approach," Journal of Chemical Physics, vol. 94, no. 11, article 7245, 1991.
[10] B. Delly, "From molecules to solids with the $\mathrm{DMol}^{3}$ approach," Journal of Chemical Physics, vol. 113, no. 18, article 7756, 2000.

[11] B. Delly, "Fast calculation of electrostatics in crystals and large molecules," The Journal of Physical Chemistry, vol. 100, no. 15, pp. 6107-6110, 1996.

[12] N. Matusuzawa, J. Seto, and D. A. Dixon, "Density functional theory predictions of second-order hyperpolarizabilities of metallocenes," The Journal of Physical Chemistry, vol. 101, no. 49, pp. 9391-9398, 1997.

[13] Y. Nishiyama, P. Langan, and H. Chanzy, "Crystal structure and hydrogen-bonding system in cellulose $\mathrm{I} \beta$ from synchrotron $\mathrm{X}$-ray and neutron fiber diffraction," Journal of the American Chemical Society, vol. 124, no. 31, pp. 9074-9082, 2002.

[14] Y. Li, M. Lin, and J. W. Davenport, "Ab initio studies of cellulose I: crystal structure, intermolecular forces, and interactions with water," Journal of Physical Chemistry C, vol. 115, no. 23, pp. 1153311539, 2011.

[15] C. Yamane, T. Aoyagi, M. Ago, K. Sato, K. Okajima, and T. Takahashi, "Two different surface properties of regenerated cellulose due to structural anisotropy," Polymer Journal, vol. 38, no. 8, pp. 819-826, 2006.

[16] Ch. Skaar, Wood-Water Relations, Springer Series in Wood Science, Springer, New York, NY, USA, 1988.

[17] A. P. Heiner and O. Teleman, "Interface between monoclinic crystalline cellulose and water: breakdown of the odd/even duplicity," Langmuir, vol. 13, no. 3, pp. 511-518, 1997.

[18] D. A. I. Goring, "Thermal softening of lignin, hemicellulose and cellulose," Pulp and Paper Magazine, vol. 64, no. 12, pp. T517T527, 1963.

[19] K. A. Dean, P. Von Allmen, and B. R. Chalamala, "Three behavioral states observed in field emission from single-walled carbon nanotubes," Journal of Vacuum Science and Technology B, vol. 17, no. 5, pp. 1959-1969, 1999.

[20] http://www.accelrys.com/products/mstudio/modeling/quantumandcatalysis/dmol3.html.

[21] A. Maiti, J. Andzelm, N. Tanpipat, and P. Von Allmen, "Effect of adsorbates on field emission from carbon nanotubes," Physical Review Letters, vol. 87, no. 15, Article ID 155502, 4 pages, 2001.

[22] A. D. Becke, "A multicenter numerical integration scheme for polyatomic molecules," The Journal of Chemical Physics, vol. 88, no. 4, pp. 2547-2554, 1988.

[23] C. Lee, W. Yang, and R. G. Parr, "Development of the ColleSalvetti correlation-energy formula into a functional of the electron density," Physical Review B, vol. 37, no. 2, pp. 785-789, 1988. 

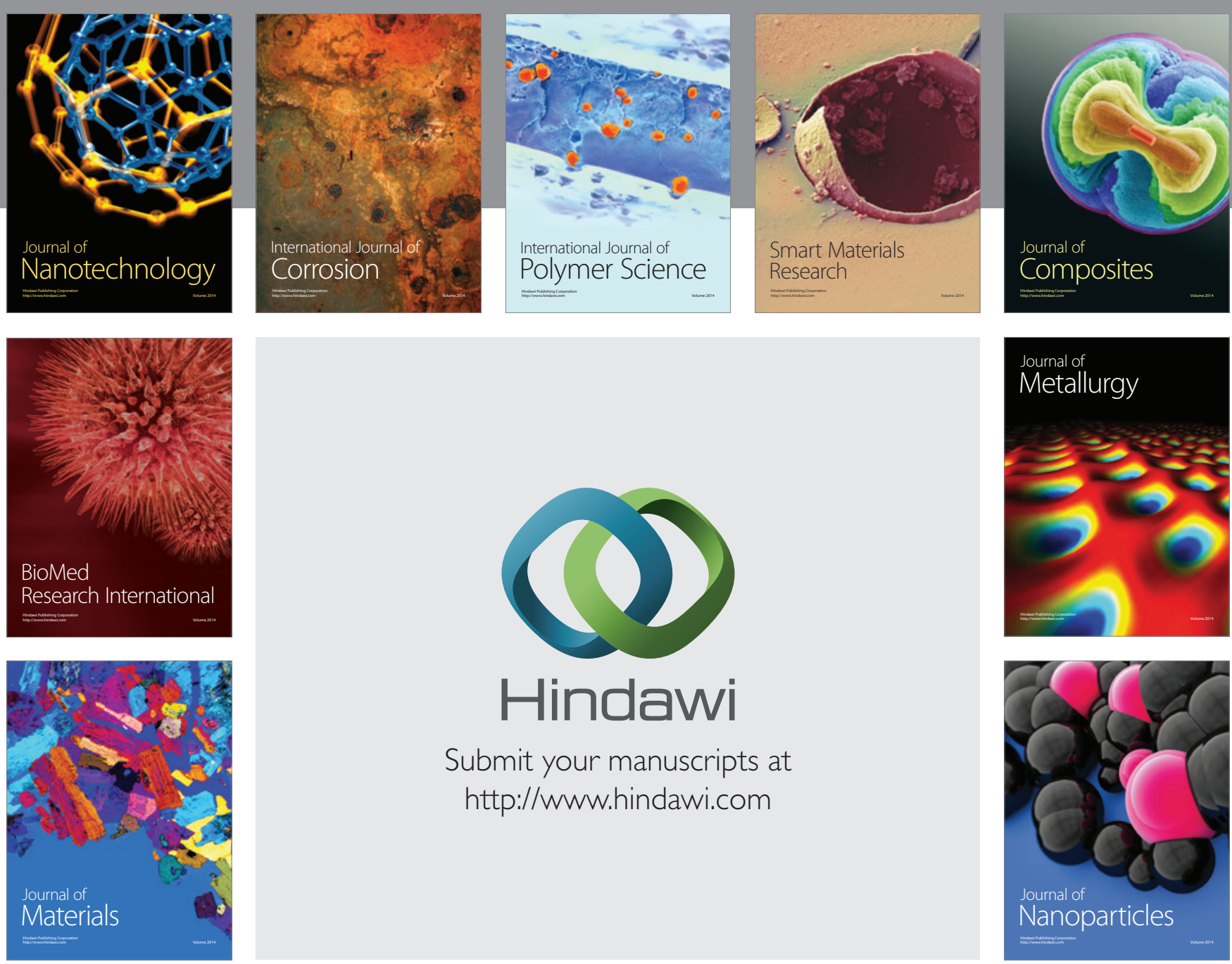

Submit your manuscripts at http://www.hindawi.com
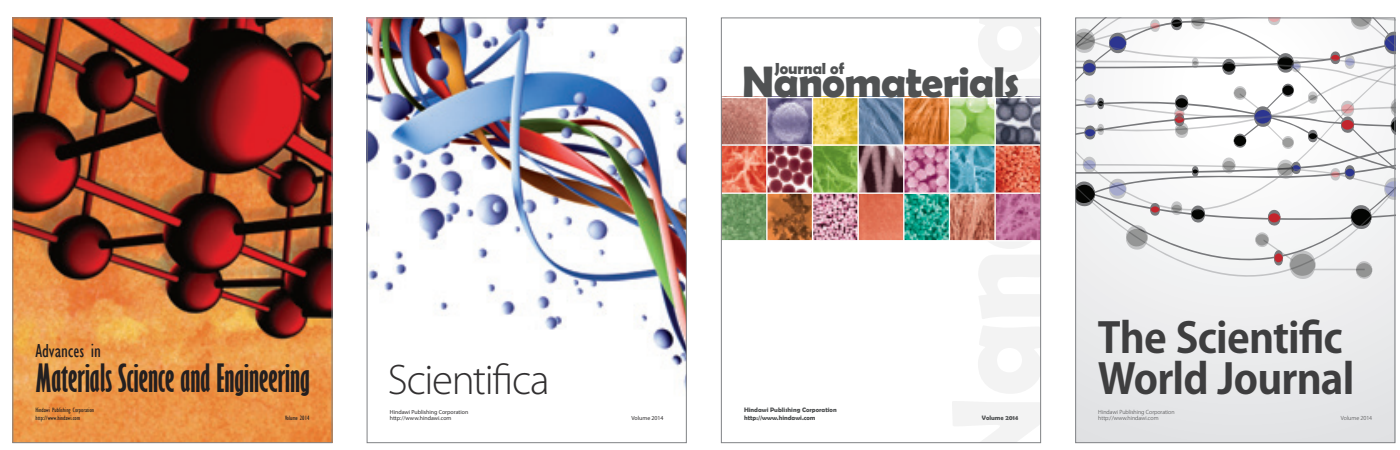

\section{The Scientific World Journal}
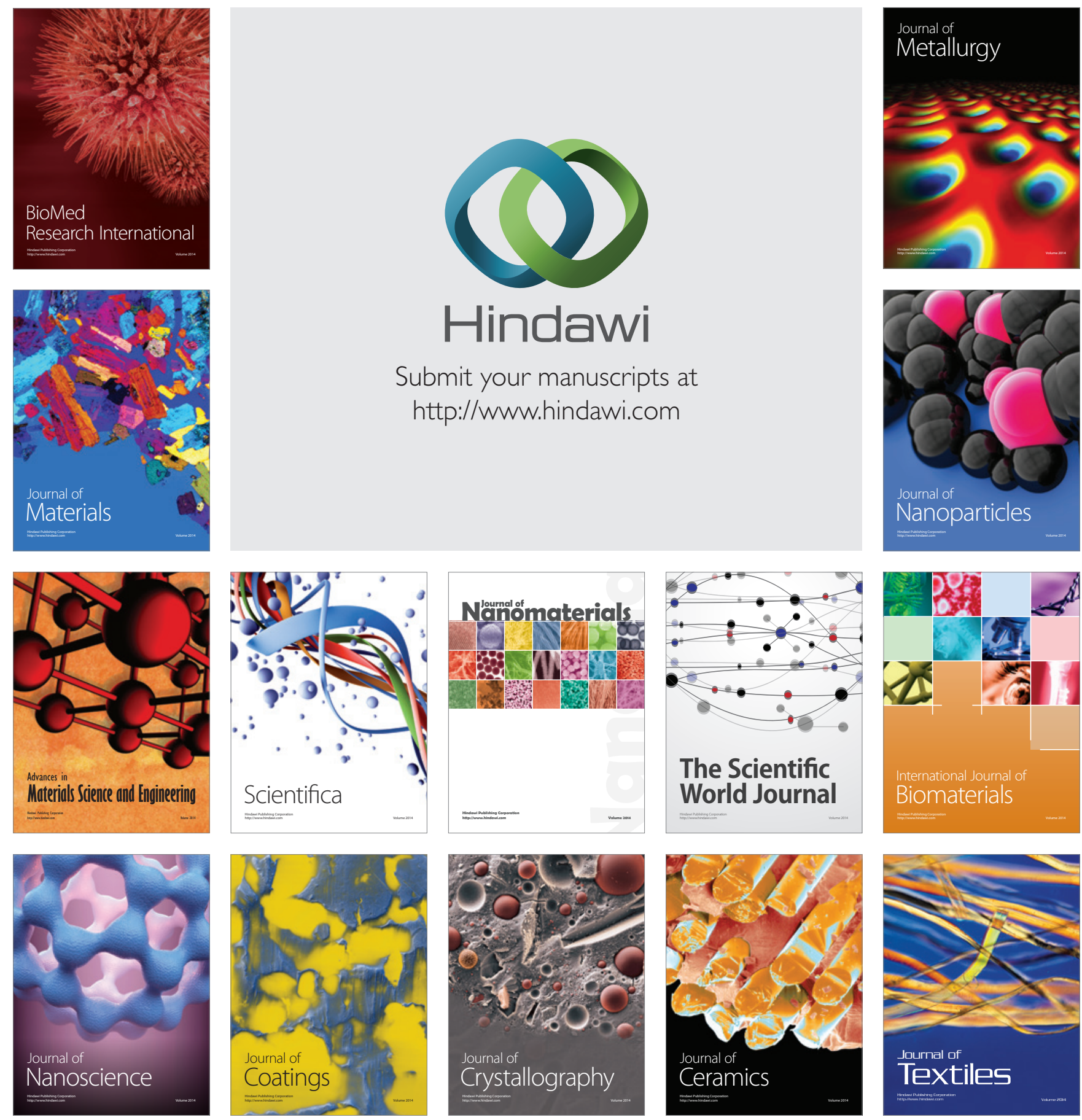